\title{
Study on Surface Morphology of Tapered Roller and Oilstone in Through-feed Super-finishing
}

\author{
Xue Jinxue ${ }^{1, a}$, Wang lina ${ }^{1, b}$ and Zhang Ying ${ }^{1, c}$ \\ ${ }^{1}$ School of Mechatronics Engineering, Henan University of Science \& Technology, Luoyang, China \\ axjx19652000@163.com, bwanglinazs@163.com, czhangypds@163.com
}

Keywords: Through-feed Super-finishing, Tapered Roller, Oilstone, Surface Morphology

Abstract. Based on the contact geometry characteristics of tapered roller and oilstone in the super-finishing process and the relative motion relation principle, the mathematical model of single abrasive trajectory on the outer surface of the oilstone was established. The model and their parameters estimation were simulated with MATLAB. The influences of the key elements on the 3D surface morphology and the surface quality of the work-piece were analyzed. The changing abrasive trajectory of the tapered roller in the super-finishing region is the principal factor that causes the dynamical variation of work-piece surface quality. The results are given as follows. With the increase of the amplitude of oilstone and the frequency of oilstone, the abrasive trajectory numbers increase accordingly. In a certain speed range, the abrasive trajectory increases with the decrease of the through-speed.

\section{Introduction}

The tapered roller is a key component of rolling bearings [1]. The shape of the roller crown is important to roller quality [2].Both theoretical analysis and experimental verification have proven that logarithmic crown is perfect [3]. The logarithmic crown has a positive effect on improving contact stress distribution of rollers and raceways and it is important for improving quality and life of rolling bearings [4,5]. Super-finishing is the most important process to machine the roller crown [6]. Yet formation mechanism of roller crown is not clear in theory. The distribution condition of oilstone abrasive is an important factor which influences the material removal rate in tapered roller super-finishing process. This paper draws lessons from trajectory model of the abrasive, bases on experimental data and parameters, and analyses contact surface topography in super-finishing area.

\section{Processing Method of the Through-feed Super-finishing}

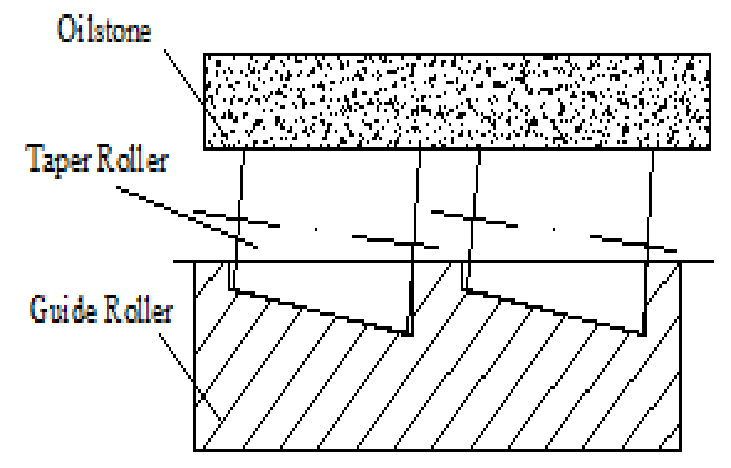

Fig.1 Through-feed super-finishing of tapered roller

Fig. 1 depicts the processing method of through-feed super-finishing of tapered rollers by oilstones. The guide roller rotates around a fixed axis and in the same direction. The rollers move along the axial and rotate by the driving force of the guide roller. A row of oilstones press on the rollers with appropriate pressures and oscillate back and forth axially. 


\section{Kinematic analysis}

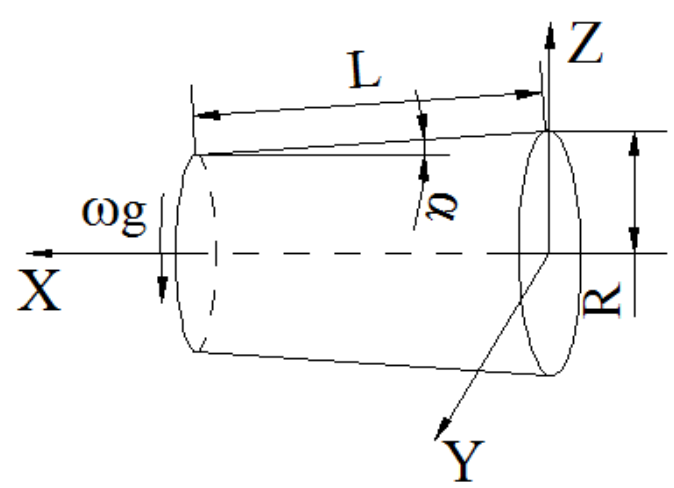

Fig.2 Analysis coordinate system of abrasive grain trajectory on tapered roller surface

Fig. 2 represents the cone equation of tapered roller, and the motion model of the abrasive particles can be established. The cone equation of the space coordinate system between the abrasive and the work-piece is as follows.

$$
\left\{\begin{array}{c}
Y^{2}+Z^{2}=(R-X \tan \alpha)^{2} \\
0 \leq X \leq L \cos \alpha
\end{array} .\right.
$$

where, $R$ represents big endian radius of work-piece, $L$ represents tapered roller cone length; $\alpha$ represents the half cone angle tapered roller, $\omega_{\mathrm{d}}$ represents rotational speed of the roller.

The velocity of the work-piece along the straight line is as follows.

$v_{d}=\omega_{d} h / 2 \pi$

where, $\omega_{a}$ represents rotational speed of the roller, $h$ represents spiral lead of the guide roller.

The rotation speed of the work-piece is as follows.

$$
v_{\mathrm{g}}=\omega_{\mathrm{g}} R
$$

It is assumed that the oscillating equation of the whetstone is as follows.

$$
Z=A \sin (2 \pi f) \text {. }
$$

By mathematical knowledge, the oilstone reciprocating oscillation speed is as follows.

$$
v_{s}=d Z / d t=2 \pi f A \cos (2 \pi f)
$$

where, $A$ represents amplitude of the oilstone reciprocating oscillation, $f$ represents the oilstone reciprocating oscillation frequency.

\section{Establishment of motion model}

Based on the relative motion analysis of the oilstone and the work-piece, the trajectory equation of any single abrasive particle on the surface of the tapered roller can be expressed at the $t$ moment with the $t$ time as the model variable. Therefore, the equation of motion trajectory of any single abrasive at the $t$ moment on the surface of the tapered roller is as follows. 


$$
\left\{\begin{array}{c}
X=v_{d} t+A \sin (2 \pi f) \\
Y=(R-X \tan \alpha) \cos \left(\omega_{g} t\right) \\
Z=(R-X \tan \alpha) \sin \left(\omega_{g} t\right)
\end{array}\right.
$$

The maximum time should be calculated to allow the abrasive to have enough time to remove the work-piece material. From the above analysis, it can be seen that the super-finishing time is the longest when the velocity is linear and uniform throughout.

$$
t_{\max }=\frac{L}{V_{\min }}=\frac{L}{v_{d}}=\frac{L}{\omega_{d}{ }^{h / 2 \pi}}
$$

\section{Simulation of surface topography of tapered roller}

Based on the above theoretical analysis and the established mathematical model, the parameter values are estimated and the trajectory of the abrasive on the work-piece surface is simulated by MATLAB programming.

The input parameters are as follows: oilstone frequency $f=30 \mathrm{~Hz}$, oilstone amplitude $A=3 \mathrm{~mm}$, big end radius of the work-piece $R=5 \mathrm{~mm}$, half cone angle of the work-piece $\alpha=2^{\circ}$, cone length $L=14 \mathrm{~mm}$, angular velocity $\omega_{\mathrm{g}}=100 \mathrm{rad} / \mathrm{s}$.

According to Eq.1, Eq.6 and Eq.7, Fig.3, Fig.4 and Fig.5 are obtained using MATLAB programming.

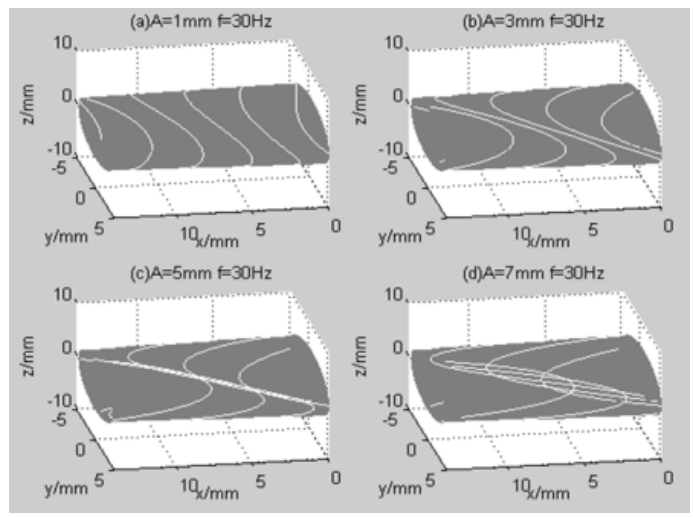

Fig.3 The influence of $A$ on abrasive trajectory

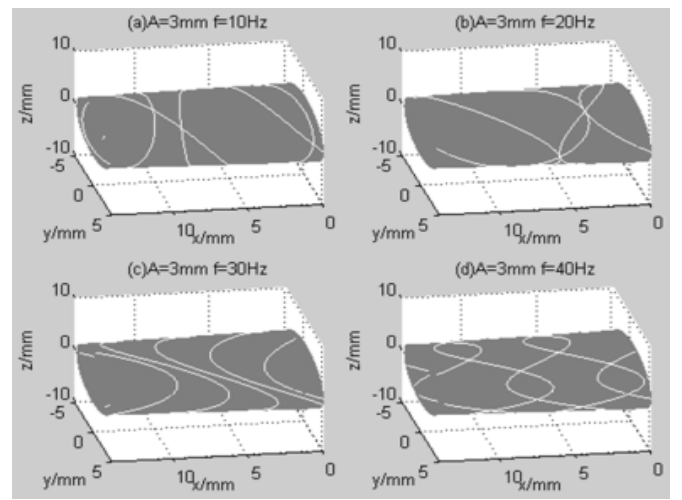

Fig.4 The influence of $f$ on abrasive trajectory

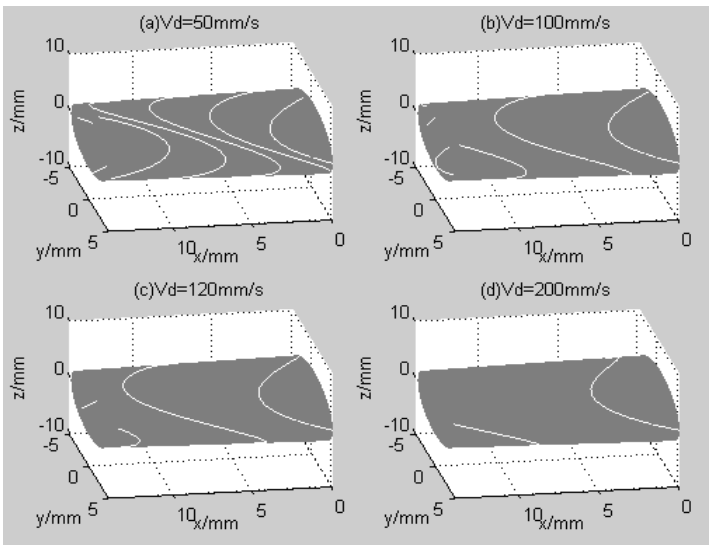

Fig.5 The influence of $v_{d}$ on abrasive trajectory

It can be seen from Fig.3 that when the other process parameters remain unchanged, the abrasive trajectory of the work-piece surface changes with the variations of the amplitude of the oilstone. 
When the $A$ increases, the cross grid density of tapered roller surface is higher, which is beneficial to storage grinding fluid and the amount of material removal on the work-piece surface is large.

It can be seen from Fig.4 that when the other process parameters remain unchanged, the abrasive trajectory of the work-piece surface changes with the variations of the frequency of the oilstone. When the $f$ increases, the abrasive trajectory increases in the unit area, and the cross reticulate increases with the decrease of the surface roughness of the material.

It can be seen from Fig. 5 that when the other process parameters remain unchanged, the abrasive trajectory of the work-piece surface changes with the variations of the linear through-feed speed $\left(v_{d}\right)$.The trajectory of abrasive on the work-piece surface decreases with the increase of the linear through-feed speed $\left(v_{d}\right)$ of the tapered roller.

From the above analysis, the number of abrasive trajectories and the number of intersecting lines determine the amount of material removal and the surface quality of the work-piece.

\section{Conclusions}

According to the analysis of the surface morphology of tapered roller and oilstone in through-feed super-finishing, we can reach some conclusions:

1) In super-finishing process, the motion trajectory of the oilstone abrasive on the surface of the work-piece is similar to the space sine curve.

2) In a certain range, the greater the amplitude of $A$ and the greater the frequency of $f$, the higher the density of the cross grid of the tapered roller surface, the larger the amount of material removal on the work-piece surface, and the smaller the roughness of the surface of the grinding surface, so as to improve the surface quality of the work-piece.

3) In the case of stable motion, with the increase of the linear through-feed speed $\left(v_{d}\right)$ of the tapered roller, the abrasive trajectory numbers decrease accordingly and the less amount of the material removal.

\section{Acknowledgements}

This research derives from the National Natural Science Foundation in China (No. 51475145).

\section{References}

[1] Xue Jinxue, Yang Baisong, Jia Songyang: Analysing Cutting Edge of Tapered Roller and Oilstone in Fixed Posture Through-feed Superfinishing. Mechanical Science and Technology for Aerospace Engineering.36-8 (2017), p. 1244-1249

[2] XUE Jin-xue, JIA Song-yang, YANG Bai-song: Grinding Analysis of Superfinishing Tapered Roller Based on Arc MRR. Coal Mine Machinery. 36-12 (2015), p. 124-126

[3] China Mechanical Engineering Society, in: Technology Roadmaps of Chinese Mechanical Engineering, edited by Science and technology of China press, Beijing (2011).

[4] CHEN Fanghua, WANG Jiugen, ZHANG Genyuan: Elastohydrodynamic Lubrication of Tapered Roller with Logarithmic Profile. JOURNAL OF MECHANICAL ENGINEERING. 47-19 (2011), p. 143-148

[5] MA Jia-ju, XU Wen, CHEN Xiao-yang: EHL Properties of Roller Contacts and Its Application, Partll: A Numerical Analysis of EHL for Engineering Logarithmic Roller. TRIBOLOGY. 20-1 (2000), p.63-66

[6] XUE Jin-xue, JIA Song-yang, YANG Bai-song: The polish properties analysis of through-feed superfinishing tapered roller. Manufacturing Automation. 38-1 (2016), p. 33-37 